https://doi.org/10.15407/dopovidi2021.03.003

УДК 517.956 .223

O.О. Мурач, https://orcid.org/0000-0001-6656-8262

I.C. Чепурухіна, https://orcid.org/0000-0002-3107-4075

Інститут математики НАН України, Київ

E-mail: murach@imath.kiev.ua, Chepurukhina@gmail.com

\title{
Еліптичні задачі з грубими крайовими даними у просторах Нікольського
}

Представлено членом-кореспондентом НАН України А.Н. Кочубеєм

Досліджено загальну еліптичну задачу, задану в обмеженій евклідовій області, з крайовими даними у просторах Нікольського низького, зокрема, від'ємного порядку. Припускається, що права частина еліптичного диференціального рівняння є інтегровною функиією. Встановлено нетеровість задачі, максимальну регуляр ність і апріорну оцінку ї узагальнених розв'язків у вказаних просторах. Дано застосування цих результатів до деяких еліптичних задач з крайовими даними, породженими гауссовим білим шумом.

Ключові слова: еліптична крайова задача, простір Нікольського, нетерів оператор, регулярність розв'язку, апріорна оцінка, білий шум.

У теорії багатовимірних крайових задач окрему увагу привертають задачі з грубими крайовими даними, тобто такими, що не є інтегровними функціями на межі (див., наприклад, [1-4]). До останніх належать функції зі степеневими особливостями, дельта-функції та їх похідні, розподіли, породжені різними випадковими процесами. Вони інтерпретуються у сенсі теорії узагальнених функцій (розподілів) як елементи деяких функціональних просторів від’ємного порядку. Дослідження таких задач є істотно складнішим порівняно з випадком достатньо регулярних крайових даних, які належать до просторів додатного порядку. Тут зразу постає питання про інтерпретацію слідів узагальнених функцій низької регулярності на межі області, де розглядається задача. Як правило, такі сліди означають за допомогою граничних переходів, апроксимуючи узагальнені функції в області досить гладкими функціями у спеціально підібраних нормах. Уведення таких норм накладає деякі умови регулярності на праву частину диференціального рівняння [1] або приводить до того, що розв’язок задачі не можна інтерпретувати як узагальнену функцію в області [2]. Додаткові складнощі виникають, коли використовують простори розв’язків, у яких множини гладких функцій не є щільними, як, наприклад, простори Нікольського низького порядку.

Ци т в ання: Мурач О.О., Чепурухіна І.С. Еліптичні задачі з грубими крайовими даними у просторах Нікольського. Допов. Наи. акад. наук Укр. 2021. № 3. С. 3-10. https://doi.org/10.15407/dopovidi2021.03.003 
Мета цієї роботи - встановити теореми про характер розв’язності (нетеровість), максимальну регулярність і апріорну оцінку розв’язків еліптичних задач з крайовими даними у просторах Нікольського низького (у тому числі від'ємного) порядку. Вона мотивована важливими застосуваннями останніх у теорії білого шуму. Саме у термінах просторів Нікольського від'ємного порядку вдалося точно (за порядком простору) охарактеризувати регулярність гауссового білого шуму на торі [5]. Це дає змогу нам отримати точний результат про регулярність розв'язків деяких еліптичних задач з крайовими умовами, породженими таким шумом.

1. Постановка задачі. Нехай $\Omega$ - обмежена область у евклідовому просторі $\mathbf{R}^{n}$, де $n \geqslant 2$. Припустимо, що ії межа $\Gamma$ є нескінченно гладким замкненим многовидом вимірності $n-1$ (причому $C^{\infty}$-структура на $\Gamma$ породжена $\mathbf{R}^{n}$ ). Розглянемо в $\Omega$ еліптичну крайову задачу вигляду

$$
\begin{aligned}
& A u=f \text { в } \Omega, \\
& B_{j} u=g_{j} \text { на } \Gamma, \quad j=1, \ldots, l .
\end{aligned}
$$

Тут $A:=A(x, D)-$ лінійний диференціальний оператор (л. д. о.) на $\bar{\Omega}:=\Omega \cup \Gamma$ парного порядку $2 l \geqslant 2$, а кожне $B_{j}:=B_{j}(x, D)-$ крайовий л. д. о. на $\Gamma$ порядку $m_{j} \leqslant 2 l-1$. Припускаємо, що всі коефіцієнти цих л. д. о. $є$ нескінченно гладкими комплекснозначними функціями, заданими на $\bar{\Omega}$ і $\Gamma$ відповідно. Нагадаємо, що еліптичність крайової задачі $(1),(2)$ означає, що л. д. о. $A$ є правильно еліптичним на $\bar{\Omega}$, а набір $B:=\left(B_{1}, \ldots, B_{l}\right)$ крайових л. д. о. задовольняє умову Лопатинського щодо $A$ на $\Gamma$.

Нехай дійсне число $p>1$. Досліджуємо властивості розв’язку $u$ крайової задачі (1), (2) у ситуації, коли крайові дані $g_{1}, \ldots, g_{l}$ належать відповідним просторам Нікольського $B_{p, \infty}^{\sigma}(\Gamma)$ низької регулярності, зокрема, коли їх порядок $\sigma<0$. При цьому припускаємо, що права частина $f$ еліптичного рівняння (1) належить до $L_{p}(\Omega)$, тобто функція $|f|^{p}$ інтегровна на $\alpha$ (відносно міри Лебега). Додержуючись [6, п. 2.3.1] і [7, п. 3.2.2], нагадаємо означення просторів Нікольського довільного дійсного порядку б. У роботі розглядаються комплексні лінійні функціональні простори.

Довільно виберемо функцію $\varphi_{0} \in C^{\infty}\left(\mathbf{R}^{n}\right)$ таку, що $\varphi_{0}(y)=1$ за умови $|y| \leqslant 1$ та $\varphi_{0}(y)=0$ за умови $|y| \geqslant 2$, де $y \in \mathbf{R}^{n}$. Для кожного номера $k \geqslant 1$ означимо функцію $\varphi_{k}(y):=\varphi_{0}\left(2^{-k} y\right)-$ $-\varphi_{0}\left(2^{1-k} y\right)$ аргументу $y \in \mathbf{R}^{n}$. Функції $\varphi_{k}$, де ціле $k \geqslant 0$, утворюють нескінченно гладке розбиття одиниці на евклідовому просторі $\mathbf{R}^{n}$.

Нехай $\sigma-$ довільне дійсне число. За означенням, лінійний простір $B_{p, \infty}^{\sigma}\left(\mathbf{R}^{n}\right)$ складається з усіх повільно зростаючих розподілів ш на $\mathbf{R}^{n}$ таких, що

$$
\left\|w, B_{p, \infty}^{\sigma}\left(\mathbf{R}^{n}\right)\right\|:=\sup _{0 \leqslant k \in \mathbf{Z}} 2^{k \sigma}\left(\int_{\mathbf{R}^{n}}\left|F^{-1}\left[\varphi_{k} F w\right]\right|^{p}(x) d x\right)^{1 / p}<\infty,
$$

де $F$ і $F^{-1}$ - відповідно пряме і обернене перетворення Фур'є. Цей простір є повним від-

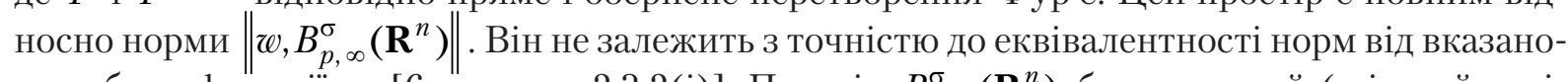
го вибору функції $\varphi_{0}\left[6\right.$, теорема 2.3.2(i)]. Простір $B_{p, \infty}^{\sigma}\left(\mathbf{R}^{n}\right)$ був уведений (в інший еквівалентний спосіб) і досліджений С.М. Нікольським для $\sigma>0$ (див. [8, с. 151; 9, с. 201] та наведені там посилання). Зауважимо, що С.М. Нікольський вибрав позначення $H_{p}^{\sigma}\left(\mathbf{R}^{n}\right)$ 
для цього простору (яке часто застосовують для просторів Соболєва). Позначення, яке використовуємо ми, загальноприйняте і свідчить про те, що шкала просторів Нікольського є специфічною частиною класу просторів Бєсова $B_{p, q}^{\sigma}\left(\mathbf{R}^{n}\right)$.

Простори Нікольського на $\Omega$ і $Г$ будуються за простором $B_{p, \infty}^{\sigma}\left(\mathbf{R}^{n}\right)$ у стандартний спосіб (див., наприклад, [7, п. 3.2.2]). А саме: лінійний простір $B_{p, \infty}^{\sigma}(\Omega)$ складається, за означенням, із звужень в область $\Omega$ усіх розподілів $w \in B_{p, \infty}^{\sigma}\left(\mathbf{R}^{n}\right)$ і наділений нормою

$$
\left\|u, B_{p, \infty}^{\sigma}(\Omega)\right\|:=\inf \left\{\left\|w, B_{p, \infty}^{\sigma}\left(\mathbf{R}^{n}\right)\right\|: w \in B_{p, \infty}^{\sigma}\left(\mathbf{R}^{n}\right), u=w \text { в } \Omega\right\},
$$

де $u \in B_{p, \infty}^{\sigma}(\Omega)$. Простір $B_{p, \infty}^{\sigma}(\Omega) \in$ повним відносно цієї норми.

Простір $B_{p, \infty}^{\sigma}(\Gamma)$ складається з усіх розподілів на $\Gamma$, які в локальних координатах на $\Gamma$ дають елементи простору $B_{p, \infty}^{\sigma}\left(\mathbf{R}^{n-1}\right)$. Дамо детальне означення. $3 C^{\infty}$-структури на многовиді Г довільно виберемо скінченний набір локальних карт $\pi_{j}: \mathbf{R}^{n-1} \leftrightarrow \Gamma_{j}$, де $j=1, \ldots, \lambda$, таких, що відкриті множини $\Gamma_{1}, \ldots, \Gamma_{\lambda}$ утворюють покриття $\Gamma$. Крім того, виберемо функції $\chi_{j} \in C^{\infty}(\Gamma)$, де $j=1, \ldots, \lambda$, які утворюють розбиття одиниці на $\Gamma$ і задовольняють умову $\operatorname{supp} \chi_{j} \subset \Gamma_{j}$. (Як звичайно, supp $\chi_{j}$ позначає замикання в топології $\Gamma$ множини усіх точок $x \in \Gamma$ таких, що $\chi_{j}(x) \neq 0$.) За означенням, лінійний простір $B_{p, \infty}^{\sigma}(\Gamma)$ складається з усіх розподілів $h$ на $\Gamma$ таких, що $\left(\chi_{j} h\right) \circ \pi_{j} \in B_{p, \infty}^{\sigma}\left(\mathbf{R}^{n-1}\right)$ для кожного номера $j \in\{1, \ldots, \lambda\}$, і наділений нормою

$$
\left\|h, B_{p, \infty}^{\sigma}(\Gamma)\right\|:=\sum_{j=1}^{\lambda}\left\|\left(\chi_{j} h\right) \circ \pi_{j}, B_{p, \infty}^{\sigma}\left(\mathbf{R}^{n-1}\right)\right\| ;
$$

тут $\left(\chi_{j} h\right) \circ \pi_{j}-$ зображення розподілу $\chi_{j} h$ у локальній карті $\pi_{j}$. Простір $B_{p, \infty}^{\sigma}(\Gamma)$ повний відносно цієї норми і з точністю до еквівалентності норм не залежить від вказаного вибору локальних карт і розбиття одиниці на Г [7, твердження 3.2.3(ii)].

Зауважимо, що множини $C^{k}(\bar{\Omega})$ і $C^{k}(\Gamma)$, де ціле $k \geqslant 0$, лежать відповідно у просторах $B_{p, \infty}^{\sigma}(\Omega)$ і $B_{p, \infty}^{\sigma}(\Gamma)$ порядку $\sigma \leqslant k$, але не є щільними у них. Останнє випливає з [9, теорема $2.3 .2(\mathrm{a})]$.

2. Основні результати. Нехай, як і раніше, $1<p<\infty$. Еліптична крайова задача (1), (2) має таку фундаментальну властивість у просторах Нікольського додатного порядку: відображення $u \mapsto(A u, B u)$, де $u \in B_{p, \infty}^{s}(\Omega)$, є нетеровим обмеженим оператором на парі просторів

$$
(A, B): B_{p, \infty}^{s}(\Omega) \rightarrow B_{p, \infty}^{s-2 l}(\Omega) \times \prod_{j=1}^{l} B_{p, \infty}^{s-m_{j}-1 / p}(\Gamma) \text { для кожного } s>2 l .
$$

Крім того, ядро цього оператора лежить у $C^{\infty}(\bar{\Omega})$ і разом з індексом не залежить від $s$ і $p$. Для регулярних еліптичних задач ця властивість доведена в [9, теорема 5.5.2(a)]. Для загальних еліптичних задач, розглянутих нами, вона міститься в результатах робіт [10, п. 3, теорема] і [11, теорема 5.2]. Позначимо через $N$ та $\alpha$ відповідно ядро та індекс оператора (3).

Принагідно нагадаємо, що лінійний обмежений оператор $T: E_{1} \rightarrow E_{2}$, де $E_{1}$ і $E_{2}-$ повні нормовані простори, називають нетеровим, якщо його ядро $\left\{x \in E_{1}: T x=0\right\}$ і коядро $E_{2} / T\left(E_{1}\right)$ скінченновимірні. Якщо цей оператор нетерів, то його область значень замкнена в $E_{2}$ i він має скінченний індекс, який, за означенням, дорівнює різниці вимірностей ядра і коядра.

Стосовно оператора (3) зауважимо, що образ $A u \in B_{p, \infty}^{s-2 l}(\Omega)$ означений у сенсі теорії розподілів для довільного $u \in B_{p, \infty}^{s}(\Omega)$, де $s-$ будь-яке дійсне число. Крім того, образ 
$B_{j} u \in B_{p, \infty}^{s-m_{j}-1 / p}(\Gamma)$ означений у сенсі теореми про сліди для кожного вказаного $u$, якщо $s>m_{j}+1 / p$ (див. [9, теорема 4.7.1(b)]); тут фіксовано номер $j \in\{1, \ldots, l\}$. Проте якщо $s \leqslant m_{j}+1 / p$, то образ $B_{j} u$ не означений коректно для довільного $u \in B_{p, \infty}^{s}(\Omega)$ навіть як деякий розподіл на $Г$. А саме: у цьому випадку відображення $u \mapsto B_{j} u$, де $u \in C^{\infty}(\bar{\Omega})$, не допускає продовження до неперервного лінійного оператора на парі просторів $B_{p, \infty}^{s}(\Omega)$ і $D^{\prime}(\Gamma)$, що випливає з $\left[9\right.$, теорема 4.7.1(d)]. Як звичайно, $D^{\prime}(\Gamma)$ позначає лінійний топологічний простір усіх розподілів на $\Gamma$.

Отже, лінійний обмежений оператор (3) не означається коректно для довільного $s<2 l$. У цьому випадку замість $B_{p, \infty}^{s}(\Omega)$ беремо вужчий простір

$$
B_{p, \infty}^{s}\left(A, L_{p}, \Omega\right)=\left\{u \in B_{p, \infty}^{s}(\Omega): A u \in L_{p}(\Omega)\right\}
$$

як область визначення оператора $(A, B)$. Цей простір наділений нормою графіка $\left\|u, B_{p, \infty}^{s}(\Omega)\right\|+$ $+\left\|A u, L_{p}(\Omega)\right\|$ і є повним відносно неї. Для довільного $u \in B_{p, \infty}^{s}\left(A, L_{p}, \Omega\right)$ коректно означений розподіл $B_{j} u \in D^{\prime}(\Gamma)$ у сенсі, який вказано нижче.

Розглянемо лінійний простір

$$
S^{\prime}\left(A, L_{p}, \Omega\right):=\left\{u \in S^{\prime}(\Omega): A u \in L_{p}(\Omega)\right\},
$$

де $S^{\prime}(\Omega)$ - лінійний топологічний простір звужень в область $\Omega$ усіх повільно зростаючих розподілів на $\mathbf{R}^{n}$. Оскільки множина $\Omega$ обмежена, то $S^{\prime}\left(A, L_{p}, \Omega\right)$ є об'єднанням усіх просторів $B_{p, \infty}^{s}\left(A, L_{p}, \Omega\right)$, де $s \in \mathbf{R}$. При цьому $B_{p, \infty}^{s}\left(A, L_{p}, \Omega\right)$ неперервно (але не щільно) вкладений у $B_{p, \infty}^{\sigma}\left(A, L_{p}, \Omega\right)$, якщо $\sigma<s$. У лінійному просторі $S^{\prime}\left(A, L_{p}, \Omega\right)$ означаємо збіжність за таким правилом: говоримо, що $u_{k} \rightarrow u$ в $S^{\prime}\left(A, L_{p}, \Omega\right)$ (при $k \rightarrow \infty$ ), якщо $u_{k} \rightarrow u$ в $B_{p, \infty}^{s}\left(A, L_{p}, \Omega\right)$ для деякого дійсного $s$. Множина $C^{\infty}(\bar{\Omega})$ щільна у просторі $S^{\prime}\left(A, L_{p}, \Omega\right)$ відносно цієї збіжності, що випливає з [3, теорема 4.25(i)] з огляду на теорему вкладення [9, теорема 4.6.2]. Для довільного $u \in S^{\prime}\left(A, L_{p}, \Omega\right)$ виберемо послідовність функцій $u_{k} \in C^{\infty}(\bar{\Omega})$ таку, що $u_{k} \rightarrow u$ в $S^{\prime}\left(A, L_{p}, \Omega\right)$. Згідно 3 [3, теорема 4.25(ii)] послідовність $\left(B_{j} u_{k}\right)_{k=1}^{\infty}$ має границю у топологічному просторі $D^{\prime}(\Gamma)$ і ця границя не залежить від вказаного вибору послідовності функцій $u_{k}$. Останню границю і беремо як $B_{j} u$. У результаті є коректно означеним лінійний неперервний оператор

$$
(A, B): S^{\prime}\left(A, L_{p}, \Omega\right) \rightarrow L_{p}(\Omega) \times\left(D^{\prime}(\Gamma)\right)^{l} .
$$

Теорема 1. Нехай $s \leqslant 2 l i 1<p<\infty$. Тоді звуження відображення (4) на простір $B_{p, \infty}^{s}\left(A, L_{p}, \Omega\right)$ є обмеженим оператором на парі просторів

$$
(A, B): B_{p, \infty}^{s}\left(A, L_{p}, \Omega\right) \rightarrow L_{p}(\Omega) \times \prod_{j=1}^{l} B_{p, \infty}^{s-m_{j}-1 / p}(\Gamma) .
$$

Оператор (5) нетерів з ядром $N$ та індексом $\alpha$ (які не залежать від $s$ i $p$ ).

Теорему 1 доречно порівняти з результатом статті [10, п. 3] про нетеровість еліптичних крайових задач у просторах Нікольського-Ройтберга довільного дійсного порядку $s$. Ці простори служать областю визначення обмеженого оператора, породженого еліптичною крайовою задачею (1), (2), і нетеровість якого встановлена у вказаній статті. Вони складаються з елементів, які не є функціями чи розподілами, якщо $s<2 l-1 / p$. Простори крайо- 
вих даних для цього оператора такі самі, як і в (5), а простір правих частин рівняння (1) складається у випадку $s<2 l$ з усіх розподілів $w \in B_{p, \infty}^{s-2 l}\left(\mathbf{R}^{n}\right)$ таких, що supp $w \subset \bar{\Omega}$. Отже, вказаний результат знаходиться поза межами теорії розподілів в області $\Omega$ у випадку, який нас цікавить.

Дослідимо локальні (впритул до частини межі області $\Omega$ ) властивості узагальнених розв’язків еліптичної крайової задачі (1), (2). Розподіл $u \in S^{\prime}(\Omega)$ називаємо узагальненим розв’язком цієї задачі з правими частинами $f \in L_{p}(\Omega)$ i $g_{1}, \ldots, g_{l} \in D^{\prime}(\Gamma)$, якщо $A u=f$ у сенсі теорії розподілів і $B_{j} u=g_{j}$ для кожного номера $j \in\{1, \ldots, l\}$ у вказаному вище сенсі для $u \in S^{\prime}\left(A, L_{p}, \Omega\right) .3$ умови $A u \in L_{p}(\Omega)$ та еліптичності л. д. о. $A$ випливає, що $\chi u \in B_{p, \infty}^{s}(\Omega)$ для кожного числа $s<2 l$ і довільної функції $\chi \in C^{\infty}(\bar{\Omega})$, яка дорівнює нулю в околі межі $\Gamma$. Втім, якщо $\chi(x) \neq 0$ на деякій частині межі, то включення $\chi u \in B_{p, \infty}^{s}(\Omega)$ буде виконуватися лише за певних умов на крайові дані $g_{1}, \ldots, g_{l}$ в околі множини $\Gamma \cap \operatorname{supp} \chi$. Якщо $s \geqslant 2 l$, то, звісно, потрібна ще деяка умова на $f$ в околі supp $\chi$, щоб гарантувати це включення. Введемо простори, у термінах яких зручно формулювати ці умови.

Нехай $U$ - відкрита підмножина простору $\mathbf{R}^{n}$ така, що $\Omega_{0}:=\Omega \cap U \neq \varnothing$ і $\Gamma_{0}:=\Gamma \cap U \neq \varnothing$. Позначимо через $B_{p, \infty}^{\sigma, \text { loc }}\left(\Omega_{0}, \Gamma_{0}\right)$, де $\sigma \in \mathbf{R}$, лінійний простір усіх розподілів $u \in S^{\prime}(\Omega)$ таких, що $\chi u \in B_{p, \infty}^{\sigma}(\Omega)$ для кожної функції $\chi \in C^{\infty}(\bar{\Omega})$, яка задовольняє умову $\operatorname{supp} \chi \subset \Omega_{0} \cup \Gamma_{0}$. Аналогічно позначимо через $B_{p, \infty}^{\sigma, \text { loc }}\left(\Gamma_{0}\right)$ лінійний простір усіх розподілів $h \in D^{\prime}(\Gamma)$ таких, що $\chi h \in B_{p, \infty}^{\sigma}(\Gamma)$ для кожної функції $\chi \in C^{\infty}(\Gamma)$, яка задовольняє умову supp $\chi \subset \Gamma_{0}$.

Теорема 2. Нехай $s \in \mathbf{R} i 1<p<\infty$. Припустимо, що розподіл $u \in S^{\prime}(\Omega) \in$ узагальненим розв'язком еліптичної крайової задачі (1), (2), праві частини якої задовольняють умови

$$
f \in L_{p}(\Omega) \cap B_{p, \infty}^{s-2 l, \text { loc }}\left(\Omega_{0}, \Gamma_{0}\right)
$$

$i g_{j} \in B_{p, \infty}^{s-m_{j}-1 / p, \text { loc }}\left(\Gamma_{0}\right)$ для кожного $j \in\{1, \ldots, l\}$. Тодi $u \in B_{p, \infty}^{s, \text { loc }}\left(\Omega_{0}, \Gamma_{0}\right)$.

Зауваження 1. Якщо $s \leqslant 2 l$, то умова (6) еквівалентна включенню $f \in L_{p}(\Omega)$ згідно з [9, теорема 4.6.1(a,b)]. Отже, у цьому випадку, висновок теореми 2 стає таким: $u \in B_{p, \infty}^{s, ~ l o c}\left(\Omega, \Gamma_{0}\right)$.

Цю теорему доповнює локальна апріорна оцінка узагальненого розв’язку $u$.

Теорема 3. Нехай $s \in \mathbf{R} i 1<p<\infty$. Припустимо, що розподіл $u \in S^{\prime}(\Omega)$ задовольняє умови теореми 2. Довільно виберемо число $r>0$ i функиї $\chi, \eta \in C^{\infty}(\bar{\Omega})$ такі, що $\operatorname{supp} \chi \subset \operatorname{supp} \eta \subset \Omega_{0} \cup \Gamma_{0} i \eta=1$ в околі $\operatorname{supp} \chi$. Тодi

$$
\begin{aligned}
& \left\|\chi u, B_{p, \infty}^{s}(\Omega)\right\| \leqslant c\left(\left\|\eta f, L_{p}(\Omega)\right\|+\left\|\eta f, B_{p, \infty}^{s-2 l}(\Omega)\right\|+\right. \\
& \left.+\sum_{j=1}^{l}\left\|\eta g_{j}, B_{p, \infty}^{s-m_{j}-1 / p}(\Gamma)\right\|+\left\|\eta u, B_{p, \infty}^{s-r}(\Omega)\right\|\right)
\end{aligned}
$$

де $c$ - деяке додатне число, яке не залежить від $u, f$ i $g_{1}, \ldots, g_{l}$.

Зауваження 2. Якщо $s \leqslant 2 l$, то в апріорній оцінці (7) можна прибрати доданок $\left\|\eta f, B_{p, \infty}^{s-2 l}(\Omega)\right\|$, а якщо $s>2 l$, то - доданок $\left\|\eta f, L_{p}(\Omega)\right\|$. Це випливає 3 [9, теорема 4.6.1(a,b)].

Зауваження 3. Оберненою до теорем 2 і 3 є така локальна властивість крайових даних у просторах Нікольського. Нехай $s \in \mathbf{R}$ i $1<p<\infty$. Припустимо, що розподіл $u \in B_{p, \infty}^{s, ~ l o c}\left(\Omega_{0}, \Gamma_{0}\right)$ $\epsilon$ узагальненим розв’язком крайової задачі (1), (2), де $f \in L_{p}(\Omega)$ i $g_{1}, \ldots, g_{l} \in D^{\prime}(\Gamma)$. Тоді $g_{j} \in B_{p, \infty}^{s-m_{j}-1 / p, \text { loc }}\left(\Gamma_{0}\right)$ для кожного $j \in\{1, \ldots, l\}$, причому 


$$
\left\|\chi g_{j}, B_{p, \infty}^{s-m_{j}-1 / p}(\Gamma)\right\| \leqslant c_{0}\left(\left\|\eta u, B_{p, \infty}^{s}(\Omega)\right\|+\left\|\eta f, L_{p}(\Omega)\right\|\right) .
$$

Тут функції $\chi$ і $\eta$ такі, як у теоремі 3 , а $c_{0}$ - деяке додатне число, яке не залежить від $u, f$ i $g_{1}, \ldots, g_{l}$. Ця властивість виконується і у випадку, коли набір $B$ крайових л. д. о. не задовольняє умову Лопатинського щодо $A$ на $\Gamma$.

Як бачимо, теореми 2 і 3 дають змогу за регулярністю крайових даних еліптичної задачі (1), (2) у просторах Нікольського (як завгодно низького порядку) встановити максимальну регулярність їі узагальненого розв’язку (у термінах ізотропних просторів). Теореми 1-3 варто порівняти з результатами нещодавно опублікованої роботи [12], в якій для деяких еліптичних з параметром крайових задач у півпросторі доведено твердження [12, теорема 6.3] про існування, єдиність, регулярність і апріорну оцінку розв’язків в анізотропних просторах, побудованих на основі ряду просторів низької регулярності, заданих на межі (зокрема, просторів Нікольського). У цій роботі не досягнуто максимальної регулярності розв’язків, як зазначає іiі автор.

Обговоримо коротко методику доведення теорем 1-3. Теорема 1 виводиться з їі аналога для $L_{p}$-просторів Соболєва в $\Omega$ за допомогою дійсної інтерполяції $\left(E_{0}, E_{1}\right)_{\theta, \infty}$ нормованих просторів $E_{0}$ і $E_{1}$. При цьому використовуються інтерполяційні формули, доведені, наприклад, в [7, п. 3.3.6], та їх версія для простору $B_{p, \infty}^{s}\left(A, L_{p}, \Omega\right)$, яка обгрунтовується подібно до [13, теорема 2]. Зазначений аналог теореми 1 доведено в [14, теорема 8.1] для регулярних еліптичних крайових задач і просторів Соболєва цілого порядку $s<0$. Для довільних еліптичних задач, розглянутих нами, він обгрунтовується подібно до [3, теореми 4.25 і 4.27]. Теореми 2 і 3 доводяться у спосіб, в ідейному плані близький до того, що використано в роботі [15, доведення теорем 4.7 і 4.13] стосовно еліптичних задач з крайовими даними низької регулярності в гільбертових узагальнених просторах Соболєва. При цьому в доведенні теореми 3 треба скористатися згаданим вище результатом статті [10, п. 3] про нетеровість еліптичних крайових задач у просторах Нікольського-Ройтберга.

3. Застосування. Розглянемо застосування теореми 1 до еліптичних задач з білим шумом у крайових умовах. Нехай $(\Theta, K, \mathbf{P})-$ імовірнісний простір, тобто $K$ - деяка $\sigma$-алгебра підмножин довільної множини $\Theta \neq \varnothing$, а $\mathbf{P}-$ деяка міра на $K$, підпорядкована умові $\mathbf{P}(\Theta)=1$. За означенням (див., наприклад, [5, с. 390]), гауссів білий шум на $Г-$ це випадкова величина $\xi: \Theta \rightarrow D^{\prime}(\Gamma)$ така, що: 1$)$ для кожного $v \in C^{\infty}(\Gamma)$ комплексна числова випадкова величина $\xi(v): \Theta \rightarrow \mathbf{C}$ має нормальний розподіл; 2) виконується рівність $\mathbf{M}\left[\xi\left(v_{1}\right) \overline{\xi\left(v_{2}\right)}\right]=$ $=C \int_{\Gamma} v_{1}(x) \overline{v_{2}(x)} d S$ для довільних функцій $v_{1}, v_{2} \in C^{\infty}(\Gamma)$ і деякого числа $C>0$, незалежного від $v_{1}$ і $v_{2}$. Тут, як звичайно, $\mathbf{M}$ - математичне сподівання, $\xi(v)-$ значення розподілу (узагальненої функції) $\xi$ на основній функції $v \in C^{\infty}(\Gamma)$, а $d S-$ елемент площі поверхні $\Gamma$.

Для гауссового білого шуму $\xi$ на торі $\mathbf{T}^{d}$ вимірності $d \geqslant 1$ доведено в [5, теорема 3.4], що

$\mathbf{P}\left\{\xi \in B_{p, \infty}^{-d / 2}\left(\mathbf{T}^{d}\right)\right\}=1$ для кожного $p \in(1, \infty)$.

Тут не можна замінити простір Нікольського на більш вузькі простори Бєсова $B_{p, q}^{-d / 2}\left(\mathbf{T}^{d}\right)$, де $p, q \in(1, \infty)$ або $p=q=\infty$, бо для них $\mathbf{P}\left\{\xi \in B_{p, q}^{-d / 2}\left(\mathbf{T}^{d}\right)\right\}=0$. Звідси випливає, що $\mathbf{P}\left\{\xi \in B_{p, \infty}^{\sigma}\left(\mathbf{T}^{d}\right)\right\}=0$, як тільки $\sigma>-d / 2$. Отже, у формулі (8) значення параметрів $\sigma=-d / 2$ і $q=\infty$ є точними, як і умова $p<\infty$. 
Припустимо, що обмежена область $\Omega \subset \mathbf{R}^{d+1}$ має межу $\Gamma=\mathbf{T}^{d}$, де $d \geqslant 1$. Розглянемо у цій області крайову задачу Діріхле для рівняння Пуассона

$$
\Delta u=f \quad \text { в } \Omega, \quad \gamma_{0} u=\xi \text { на } \quad \mathbf{T}^{d},
$$

де $f \in L_{p}(\Omega)$ для деякого $p \in(1, \infty)$, а $\xi-$ гауссів білий шум на торі $\mathbf{T}^{d}$. Тут $\Delta-$ оператор Лапласа, а $\gamma_{0}-$ крайовий оператор (порядку нуль), який кожній функції $u \in C^{\infty}(\bar{\Omega})$ ставить у відповідність їі слід (тобто звуження) на $\mathbf{T}^{d}$ i продовжується єдиним чином за допомогою граничного переходу до неперервного лінійного оператора на парі просторів $S^{\prime}\left(A, L_{p}, \Omega\right)$ і $D^{\prime}\left(\mathbf{T}^{d}\right)$. Розглянута задача $\epsilon$ еліптичною крайовою задачею вигляду $(1)$, (2), де $l=1, m_{1}=0, N=\{0\}$ і $\alpha=0$.

3 теореми 1 і властивості (8) випливає такий результат:

Теорема 4. Для $\mathbf{P}$-майже всіх $\omega \in \Theta$ крайова задача (9) має єдиний узагальнений розв'язок $и(\omega, \cdot)$ класу $B_{p, \infty}^{1 / p-d / 2}(\Omega)$. Він задовольняє апріорну оцінку

$$
\left\|u(\omega, \cdot), B_{p, \infty}^{1 / p-d / 2}(\Omega)\right\| \leqslant c\left(\left\|f, L_{p}(\Omega)\right\|+\left\|\xi(\omega), B_{p, \infty}^{-d / 2}\left(\mathbf{T}^{d}\right)\right\|\right)
$$

де $c$ - деяке додатне число, яке не залежить від $f$, $\xi$ і $\omega$.

Цей результат є точним за порядком простору Нікольського в $\Omega$. Використання просторів Бєсова $B_{p, q}^{\sigma}$ з показником $q<\infty$ не дає змоги отримати подібний точний результат, тобто досягти граничного значення $\sigma=1 / p-d / 2$. У роботі [15, висновок 8.4] його було досягнуто для деяких гільбертових узагальнених просторів Соболєва (випадок $p=2$ ), але висновок теореми 4 є більш сильним з огляду на включення у них просторів Нікольського, використаних у цій теоремі.

\section{ЦИТОВАНА ЛІТЕРАТУРА}

1. Лионс Ж.-Л., Мадженес Э. Неоднородные граничные задачи и их приложения. Москва: Мир, 1971. 372 с.

2. Roitberg Ya. Elliptic boundary value problems in the spaces of distributions. Dordrecht: Kluwer Acad. Publ., 1996. xii+415 p.

3. Mikhailets V.A., Murach A.A. Hörmander spaces, interpolation, and elliptic problems. Berlin, Boston: De Gruyter, 2014. xii+297 p.

4. Behrndt J., Hassi S., de Snoo H. Boundary value problems, Weyl functions, and differential operators. Cham: Springer, 2020. $772 \mathrm{p}$.

5. Veraar M. Regularity of Gaussian white noise on the $d$-dimensional torus. Banach Center Publ. 2011. 95. P. 385-398. https://doi.org/10.4064/bc95-0-24

6. Triebel H. Theory of function spaces. II. Basel: Birkhäuser, 1992. viii+370 p.

7. Трибель Х. Теория функциональных пространств. Москва: Мир, 1986. 447 с.

8. Никольский С.М. Приближение функций многих переменных и теоремы вложения. Москва: Наука, 1977. $456 \mathrm{c}$.

9. Трибель Х. Теория интерполяции, функциональные пространства, дифференциальные операторы. Москва: Мир, 1980. 664 с.

10. Мурач А.А. Эллиптические краевые задачи в полных шкалах пространств типа Никольского. Укр. мат. журн. 1994. 46, № 12. С. 1647-1654.

11. Johnsen J. Elliptic boundary problems and the Boutet de Monvel calculus in Besov and Triebel-Lizorkin spaces. Math. Scand. 1996. 79, № 1. P. 25-85. https://doi.org/10.7146/math.scand.a-12593

12. Hummel F. Boundary value problems of elliptic and parabolic type with boundary data of negative regularity. J. Evol. Equ. 2021. https://doi.org/10.1007/s00028-020-00664-0 
13. Kasirenko T., Mikhailets V., Murach A. Sobolev-like Hilbert spaces induced by elliptic operators. Complex Anal. Oper. Theory. 2019. 13, № 3. P. 1431-1440. https://doi.org/10.1007/s11785-018-00886-8

14. Lions J.-L., Magenes E. Problèmes aux limites non homogénes. VI. J. Anal. Math. 1963. 11. P. 165-188. https://doi.org/10.1007/BF02789983

15. Anop A., Denk R., Murach A. Elliptic problems with rough boundary data in generalized Sobolev spaces. Commun. Pure Appl. Anal. 2021. 20, № 2. P. 697-735. https://doi.org/10.3934/cpaa.2020286

Надійшло до редакції 17.03.2021

\section{REFERENCES}

1. Lions, J.-L. \& Magenes, E. (1972). Non-homogeneous boundary-value problems and applications, vol. I. Berlin: Springer.

2. Roitberg, Ya. (1996). Elliptic boundary value problems in the spaces of distributions. Dordrecht: Kluwer Acad. Publ.

3. Mikhailets, V. A. \& Murach, A. A. (2014). Hörmander spaces, interpolation, and elliptic problems. Berlin, Boston: De Gruyter.

4. Behrndt, J., Hassi, S. \& de Snoo, H. (2020). Boundary value problems, Weyl functions, and differential operators. Cham: Springer.

5. Veraar, M. (2011). Regularity of Gaussian white noise on the d-dimensional torus. Banach Center Publ., 95, pp. 385-398. https://doi.org/10.4064/bc95-0-24

6. Triebel, H. (1992). Theory of function spaces. II. Basel: Birkhäuser.

7. Triebel, H. (1983). Theory of function spaces. Basel: Birkhäuser.

8. Nikol'skii, S. M. (1977). Approximation of functions of several variables and imbedding theorems. 2nd ed. Moscow: Nauka (in Russian.)

9. Triebel, H. (1995). Interpolation theory, function spaces, differential operators. 2nd ed. Heidelberg: Johann Ambrosius Barth.

10. Murach, A. A. (1994). Elliptic boundary value problems in complete scales of Nikol'skii-type spaces. Ukr. Math J., 46, No. 12, pp. 1827-1835. https://doi.org/10.1007/BF01063170

11. Johnsen, J. (1996). Elliptic boundary problems and the Boutet de Monvel calculus in Besov and TriebelLizorkin spaces. Math. Scand., 79, No. 1, pp. 25-85. https://doi.org/10.7146/math.scand.a-12593

12. Hummel, F. (2021). Boundary value problems of elliptic and parabolic type with boundary data of negative regularity. J. Evol. Equ. https://doi.org/10.1007/s00028-020-00664-0

13. Kasirenko, T., Mikhailets, V. \& Murach, A. (2019). Sobolev-like Hilbert spaces induced by elliptic operators. Complex Anal. Oper. Theory, 13, No. 3, pp. 1431-1440. https://doi.org/10.1007/s11785-018-00886-8

14. Lions, J.-L. \& Magenes, E. (1963). Problèmes aux limites non homogénes. VI. J. d'Analyse Math., 11, pp. 165188. https://doi.org/10.1007/BF02789983

15. Anop, A., Denk, R. \& Murach, A. (2021). Elliptic problems with rough boundary data in generalized Sobolev spaces. Commun. Pure Appl. Anal., 20, No. 2, pp. 697-735. https://doi.org/10.3934/cpaa.2020286

Received 17.03.2021

A.A. Murach, https://orcid.org/0000-0001-6656-8262

I.S. Chepurukhina, https://orcid.org/0000-0002-3107-4075

Institute of Mathematics of the NAS of Ukraine, Kyiv

E-mail: murach@imath.kiev.ua, Chepurukhina@gmail.com

\section{ELLIPTIC PROBLEMS WITH ROUGH BOUNDARY DATA IN NIKOLSKII SPACES}

We investigate a general elliptic problem given in a bounded Euclidean domain with boundary data in Nikolskii spaces of low, specifically, negative order. The right-hand side of the elliptic differential equation is supposed to be an integrable function. We establish the Fredholm property of the problem, maximal regularity, and a priori estimate of its generalized solutions in the spaces indicated. We give an application of these results to some elliptic problems with boundary conditions induced by a Gaussian white noise.

Keywords: elliptic boundary-value problem, Nikolskii space, Fredholm operator, regularity of solution, a priori estimate, white noise. 